\title{
Concurrent Implementation of the Optimal Incremental Approximation Method for the Adaptive and Meshless Solution of Differential Equations
}

\author{
MICHAEL KOKKOLARAS* \\ Department of Mechanical Engineering, University of Michigan, Ann Arbor, MI, USA \\ email: mk@umich.edu
}

ANDREW J. MEADE, JR.

Department of Mechanical Engineering and Materials Science, Rice University, Houston, TX, USA

email:meade@rice.edu

BORIS ZELDIN

Software Engineer, Vignette Corporation, Boston, MA, USA

email: bzeldin@email.msn.com

Received July 18, 2001; Revised May 22, 2003; Accepted June 17, 2003

Abstract. The optimal incremental function approximation method is implemented for the adaptive and meshless solution of differential equations. The basis functions and associated coefficients of a series expansion representing the solution are selected optimally at each step of the algorithm according to appropriate error minimization criteria. Thus, the solution is built incrementally. In this manner, the computational technique is adaptive in nature, although a grid is neither built nor adapted in the traditional sense using a posteriori error estimates. Since the basis functions are associated with the nodes only, the method can be viewed as a meshless method. Variational principles are utilized for the definition of the objective function to be extremized in the associated optimization problems. Complicated data structures, expensive remeshing algorithms, and systems solvers are avoided. Computational efficiency is increased by using low-order local basis functions and the parallel direct search (PDS) optimization algorithm. Numerical results are reported for both a linear and a nonlinear problem associated with fluid dynamics. Challenges and opportunities regarding the use of this method are discussed.

Keywords: meshless methods, adaptive solutions, incremental approximation, parallel optimization

\section{Introduction}

The purpose of adaptive grid optimization techniques in computational mechanics is to generate an optimal node distribution according to some objective. The common characteristic of these techniques is that a grid is generated and a solution is found. Based on the solution, the grid is adapted and regenerated until the objective is achieved. Typical objectives include minimization of the total potential energy of the system (Oliveira, 1971) or 
homogeneous distribution of the local approximation error over the elements (Demkowicz and Oden, 1986).

Diaz et al. (1983) considered the minimization of an estimated upper bound on the potential energy of the approximate solution. They classified their optimal node distribution technique as a relocation $(r)$ adaptive method. Using finite element error analysis (Johnson, 1990), the error $\epsilon$ between the exact solution $u(\bar{x})$ and its approximation is bounded by

$$
\|\epsilon\|_{H^{m}} \leq C_{\delta} h^{\mu}\|u(\bar{x})\|_{H^{s}} \approx C_{\delta} N^{-\mu}\|u(\bar{x})\|_{H^{s}},
$$

where $\bar{x}$ is the vector of independent variables, $\mu=\min (p+1-m, s-m), C_{\delta}$ is some positive constant, $p$ is the polynomial order of the used basis functions, $h$ is the grid spacing, $N \approx h^{-1}$ is the number of used basis functions, and $s$ and $m$ are the order of the Hilbert space norms in the domain of interest. In $h$-methods, the grid spacing $h$ is reduced through the introduction of additional basis functions to improve the quality of the solution. In $p$-methods, the polynomial order $p$ of the basis functions is increased while $h-p$ methods combine the introduction of additional basis functions with an increase in the order of existing and/or introduced bases. In the $r$-method, the location of the grid nodes is adjusted to increase the accuracy by decreasing the value of the constant $C_{\delta}$.

It is well-known that most of the computational time required by codes that utilize adaptive techniques is spent on grid regeneration (Hassan et al., 1992; Eiseman et al., 1992). Oden (1992) stated that the major challenges to adaptive methods in computational mechanics include:

1. The use of unstructured meshes and their resulting elaborate and complicated data structures.

2. The necessity of explicit or iterative solution techniques due to the poor performance of direct solvers on dynamically evolving unstructured meshes.

3. Stability issues of the associated numerical schemes stemming from the continuous changes in the data structures and polynomial order of the bases.

4. The computational overhead of the error estimation and the adaptation process.

Motivated by similarities observed in artificial neural network algorithms and adaptive grid optimization in computational mechanics, Meade et al. (1997) formulated the concept of sequential function approximation (SFA) for the solution of differential equations. Using the closely related methods of weighted residuals and variational principles, appropriate optimization problems were formulated, and incrementally built solutions were obtained for one- and two-dimensional boundary value problems involving linear self-adjoint differential operators associated with homogeneous Dirichlet boundary conditions. We refer to the utilization of the SFA concept for building optimal approximations to solutions of differential equations incrementally as the optimal incremental approximation (OIA) method.

Since the above development, so-called meshless methods have attracted a good deal of attention because many computational mechanics problems (e.g., simulation of manufacturing 
processes or crack propagation) are not well suited for the traditional finite element, finite volume, or finite difference methods (Belytschko et al., 1996). Meshless methods attempt to construct the approximate solutions entirely in terms of nodes.

In this paper, a concurrent implementation of the OIA method (presented in the next section) is applied to a linear nonself-adjoint problem (one-dimensional convection-diffusion equation, Section 3), and a nonlinear problem (two-dimensional velocity potential equation, Section 4) with general boundary conditions. The efficient adaptive nature of the proposed computational technique is illustrated by means of the numerical results. Since the obtained solutions depend on basis functions with compact domains of support that are associated solely with nodes, the OIA method can be viewed as a meshless method. A grid is neither built nor adapted. In this manner, the OIA method features the advantages of adaptive techniques without sharing their drawbacks. Generalization and possible extension issues are addressed in Section 5. Conclusions are drawn in Section 6.

\section{The OIA method}

The algorithm for optimal incremental approximation is based on the artificial neural network algorithm proposed in Jones $(1990,1992)$ and Barron (1993). The solution $u(\bar{x})$ of a problem of the general form $\mathcal{H}[u]-f=0$, where $\mathcal{H}[\cdot]$ is a general differential operator, is approximated by the series expansion

$$
u(\bar{x}) \approx u_{N}^{a}(\bar{x})=u_{0}+\sum_{j=1}^{N} c_{j} \Phi_{j}\left(\bar{x}, \bar{q}_{j}\right),
$$

where $c_{j}$ and $\Phi_{j}$ denote coefficients and basis functions, respectively. At the $N$-th step of the algorithm, $c_{j}$ and $\Phi_{j}\left(\bar{x}, \bar{q}_{j}\right)$ are held fixed for $j=1, \ldots, N-1$, and the parameters $\bar{q}_{N}$ of the function $\Phi_{N}$ and the associated coefficient $c_{N}$ are determined by solving the nonlinear optimization problem

$$
\min _{\bar{q}_{N}, c_{N}} E\left(u_{N}^{a}\right)
$$

where $E(\cdot)$ is some appropriate functional, for example, the variational principle associated with the problem $\mathcal{H}[u]-f=0$. Note that problem (3) may be subject to general equality and/or inequality constraints, e.g., bounds on the parameters of the basis functions.

The term $u_{0}$ in Eq. (2) denotes some initial approximation such as a previously obtained low-fidelity numerical solution of the problem and/or an appropriate additional series expansion necessary for the treatment of boundary conditions (e.g., non-homogeneous Dirichlet boundary conditions). This additional series expansion may involve different type(s) of functions than the ones used in the series expansion built by the proposed method. In some cases, as in the presence of Neumann boundary conditions, it may be necessary to extend the computational domain and satisfy the boundary conditions by means of the basis functions included in the series expansion built by the OIA method. This is demonstrated in the nonlinear application considered in this paper. 
With respect to the disadvantages outlined in Oden (1992), note that there is no orthogonality requirement on the basis functions, neither are there any restrictions on their type or their distribution over the domain of interest. A broad class of functions (low-order polynomials, $B$-splines, and radial basis functions, etc.) can be used and overlapping is possible. Basis functions typically have a compact domain of support and are associated solely with nodes. In this sense, additional basis functions change the computational "grid" as needed. As a result, an $r$-type adaptive grid is created virtually without the need for remeshing. It is matrix-free and can be concentrated in areas with high gradients or any other desired regions. The adaptive grid, defined by the location of the optimally determined basis function parameters, evolves with the solution and is not based on a posteriori error estimates. The computational effort associated with a posteriori error estimation and respective regridding is eliminated.

The computational efficiency of the method depends on the cost of solving the nonlinear programming problem (3) at each step of the algorithm. The size of this optimization problem is kept low by solving for one basis at a time. Although any appropriate gradient-based nonlinear programming algorithm can be used to solve the optimization problems associated with the applications considered in this paper, we chose the parallel direct search (PDS) algorithm (Dennis and Torczon, 1991) since it does not require evaluation or estimation of derivatives, a feature that can be quite useful for many engineering problems where derivatives are either not available or too noisy when computed numerically. Moreover, PDS was implemented by our collaborator David Serafini to run on a parallel computer (an IBM SP2 located at the Center for Research on Parallel Computation (CRPC) at Rice University) by means of the Message-Passing Interface (MPI). The necessary evaluations of the functional to be extremized are quite inexpensive and are distributed among four processors. A major advantage of PDS is that it is fully scalable, that is, it can exploit additional processors with the highest efficiency.

Variational principles are employed to define appropriate objective function(al)s, and numerical stability issues are limited to the nonlinear optimization process. Note that without an orthogonality requirement on the bases, the optimization problem size in the OIA method is small compared to finite elements and finite volume where the coefficients and all of the bases for an adaptive scheme must be calculated simultaneously.

\section{Linear application: The convection-diffusion equation}

A mathematical model of adequate fidelity for the steady-state balance between fluid convection and diffusion is given by the ordinary differential equation

$$
-\alpha \frac{\mathrm{d}^{2} u}{\mathrm{~d} x^{2}}+\beta \frac{\mathrm{d} u}{\mathrm{~d} x}=0 \quad \text { with } \alpha, \beta>0,0 \leq x \leq 1,
$$

where $\alpha$ and $\beta$ represent the viscosity and convection coefficients, respectively. For the boundary conditions $u(0)=0$ and $u(1)=1$, the exact solution representing velocity is given by $u(x)=\frac{e^{\beta x / \alpha}-1}{e^{\beta / \alpha}-1}$ and is characterized by the presence of a boundary layer adjacent to the surface at $x=1$ (Fletcher, 1988). For many flow problems dissipation is significant only in 
the region of the boundary layer. Therefore, numerical solutions are often oscillatory when the dependent variable exhibits large gradients across the boundary layer. The cell Reynolds number, defined in analogy to the freestream Reynolds number by $\operatorname{Re}_{c e l l}=\beta \Delta x / \alpha$, plays a significant role in the accuracy and stability of the numerical solution in finite difference methods. In this regard, Eq. (4) poses a challenging numerical problem.

Variational principles are readily available for problems that are governed by linear self-adjoint differential operators and are associated with homogeneous Dirichlet boundary conditions. For linear nonself-adjoint operators and non-homogeneous Dirichlet boundary conditions it is possible to derive variational principles by transforming the operators appropriately (Zienkiewcz and Taylor, 1989; Finlayson, 1972). The variational principle associated with the problem of Eq. (4) is derived in Kokkolaras (1997) as

$$
E=\int_{0}^{1} e^{-\frac{\beta}{\alpha} x}\left(\frac{\mathrm{d} u}{\mathrm{~d} x}\right)^{2} \mathrm{~d} x
$$

\subsection{Numerical procedure}

The function $u(x)$ that minimizes the functional of Eq. (5) satisfies the associated Euler-Lagrange equation given in Eq. (4) and the boundary conditions. The series expansion used to approximate $u(x)$ is

$$
u(x) \approx u_{N}^{a}(x)=x+\sum_{j=1}^{N} c_{j} \Phi_{j}\left(x, \bar{q}_{j}\right)=u_{N-1}^{a}(x)+c_{N} \Phi_{N}\left(x, \bar{q}_{N}\right) .
$$

The term $c_{0} \Phi_{0}=x$ is included in order to satisfy the boundary conditions since all other basis functions $\left(\Phi_{j}, j=1, \ldots, N\right)$ are defined to vanish at the boundaries of the computational domain. After introducing this series expansion into Eq. (5), the optimization variables vector $\bar{q}_{N}^{\prime}=\left(\bar{q}_{N}, c_{N}\right)^{\mathrm{T}}$ can be defined and determined using PDS. However, the size of this nonlinear optimization problem can be further reduced. By substituting Eq. (6) into Eq. (5) and requiring the derivative of $E$ with respect to $c_{N}$ to vanish, the following expression is obtained:

$$
\int_{0}^{1} e^{-\frac{\beta}{\alpha} x} \frac{\mathrm{d} u_{N}^{a}}{\mathrm{~d} x} \frac{\mathrm{d} \Phi_{N}}{\mathrm{~d} x} \mathrm{~d} x=0
$$

Solving for the coefficient $c_{N}$ yields

$$
\begin{aligned}
c_{N}= & {\left[\int_{0}^{1} e^{-\frac{\beta}{\alpha} x} \frac{\mathrm{d} \Phi_{N}}{\mathrm{~d} x} \frac{\mathrm{d} \Phi_{N}}{\mathrm{~d} x} \mathrm{~d} x\right]^{-1} } \\
& \times\left[-\int_{0}^{1} e^{-\frac{\beta}{\alpha} x} \frac{\mathrm{d} \Phi_{N}}{\mathrm{~d} x} \mathrm{~d} x-\sum_{j=1}^{N-1} c_{j} \int_{0}^{1} e^{-\frac{\beta}{\alpha} x} \frac{\mathrm{d} \Phi_{j}}{\mathrm{~d} x} \frac{\mathrm{d} \Phi_{N}}{\mathrm{~d} x} \mathrm{~d} x\right] .
\end{aligned}
$$


Readily parameterized piecewise linear "hat" functions ( $B_{1}$-splines) were employed as the basis functions. $B_{1}$-splines are defined as

$$
\Phi(x)= \begin{cases}\left(x-\left(x_{M}-\Delta x_{l}\right)\right) / \Delta x_{l} & \text { if } x_{M}-\Delta x_{l} \leq x \leq x_{M} \\ \left(\left(x_{M}+\Delta x_{r}\right)-x\right) / \Delta x_{r} & \text { if } x_{M} \leq x \leq x_{M}+\Delta x_{r} \\ 0 & \text { otherwise }\end{cases}
$$

where the parameters $x_{M}, \Delta x_{l}$, and $\Delta x_{r}$ denote the location of the center of the function, its width to the left, and its width to the right, respectively. The optimization variables vector is $\bar{q}_{N}=\left(x_{M}, \Delta x_{l}, \Delta x_{r}\right)_{N}^{\mathrm{T}}$ and can be computed by solving the optimization problem

$$
\begin{aligned}
& \min _{\bar{q}_{N}} E\left(u_{N}^{a}\right) \\
& \text { subject to } 0 \leq x_{M}-\Delta x_{l} \leq x_{M} \leq x_{M}+\Delta x_{r} \leq 1,
\end{aligned}
$$

where

$$
\begin{aligned}
E\left(u_{N}^{a}\right)= & \int_{0}^{1} e^{-\frac{\beta}{\alpha} x}\left(1+\sum_{j=1}^{N-1} c_{j} \frac{\mathrm{d} \Phi_{j}}{\mathrm{~d} x}+\left[\int_{0}^{1} e^{-\frac{\beta}{\alpha} x} \frac{\mathrm{d} \Phi_{N}}{\mathrm{~d} x} \frac{\mathrm{d} \Phi_{N}}{\mathrm{~d} x} \mathrm{~d} x\right]^{-1}\right. \\
& \left.\times\left[-\int_{0}^{1} e^{-\frac{\beta}{\alpha} x} \frac{\mathrm{d} \Phi_{N}}{\mathrm{~d} x} \mathrm{~d} x-\sum_{j=1}^{N-1} c_{j} \int_{0}^{1} e^{-\frac{\beta}{\alpha} x} \frac{\mathrm{d} \Phi_{j}}{\mathrm{~d} x} \frac{\mathrm{d} \Phi_{N}}{\mathrm{~d} x} \mathrm{~d} x\right] \frac{\mathrm{d} \Phi_{N}}{\mathrm{~d} x}\right)^{2} \mathrm{~d} x
\end{aligned}
$$

Once the basis functions parameters $\bar{q}_{N}$ have been determined, the associated coefficient $c_{N}$ can be computed directly from Eq. (8).

\section{2. $\quad$ Numerical results}

Equation (4) was solved for the coefficient ratio $\beta / \alpha=20$. The approximations obtained after determining optimally the parameters of one (top left), two (top right), three (bottom left), and ten (bottom right) basis functions and the associated coefficients are compared to the exact solution in figure 1. The agreement between the exact and approximate solutions within the boundary layer, characterized by a large gradient of the solution, is quite satisfactory. Acknowledging that finite elements with $B_{1}$ splines can mimic finite difference schemes using specific quadrature rules (Fletcher, 1982), figure 1 illustrates the low number of bases required by the OIA method relative to results reproduced from Fletcher (1988) in figure 2. In addition, solutions obtained at any step of the algorithm do not exhibit instabilities (oscillations) as opposed to solutions obtained by some finite difference schemes. The same oscillations occur when applying the Bubnov-Galerkin finite element method to the original differential equation using coarse uniform grids and linear Lagrangian shape functions. 

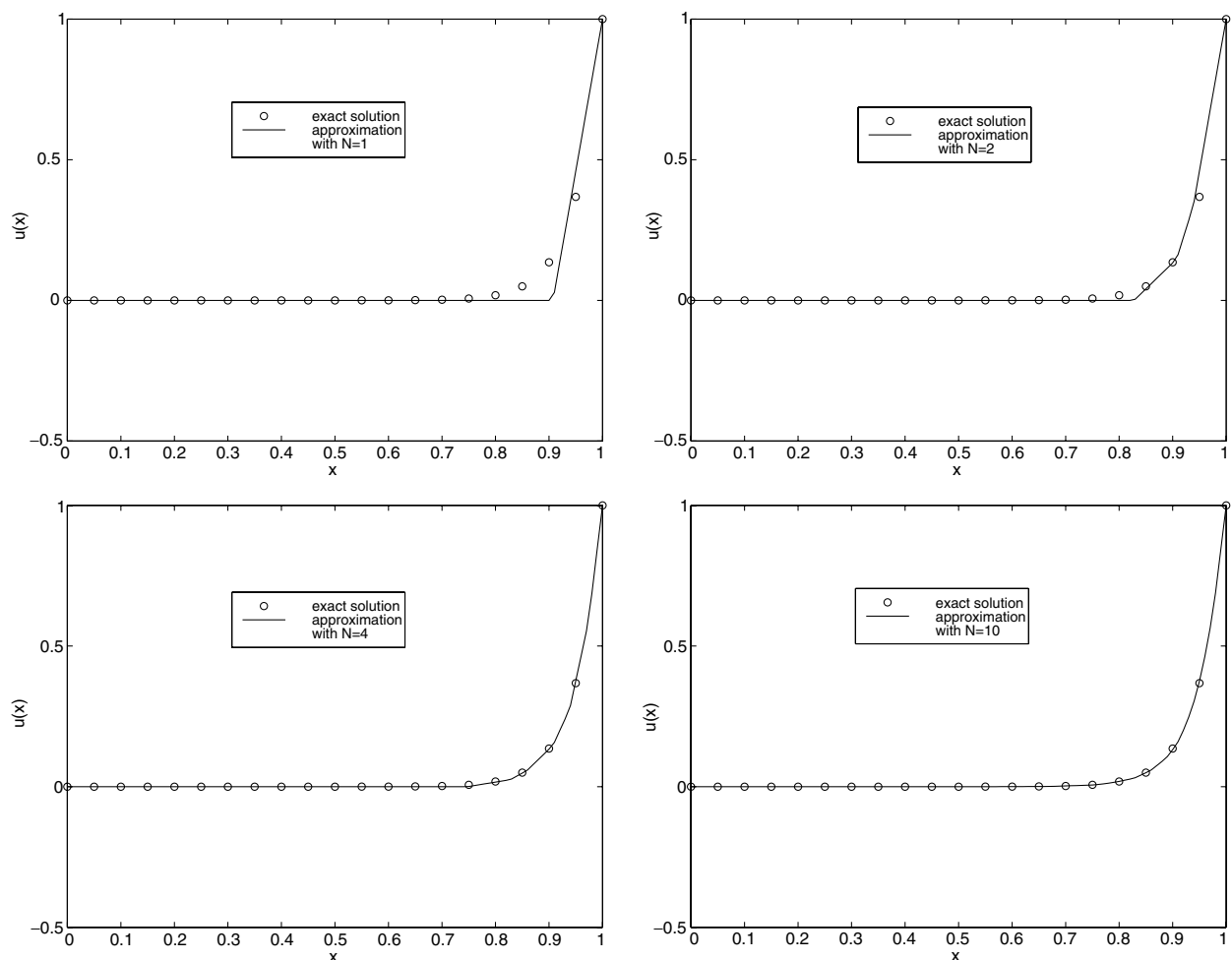

Figure 1. One-dimensional convection-diffusion. The evolution of the approximate solution with increasing number $N$ of basis functions using the OIA method compared to the exact solution.
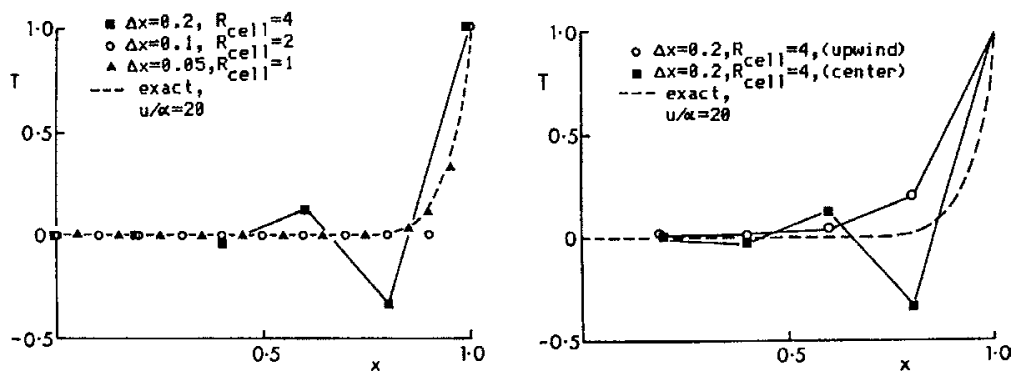

Figure 2. One-dimensional convection-diffusion. Solutions from different schemes of the finite differences method (reproduced from Fletcher (1988)).

Figure 3 (left) compares the RMS error convergence rate obtained by the Galerkin method using linear Lagrangian shape functions on a uniform grid to that obtained by the optimal incremental approximation method. The RMS error is directly proportional to the discrete $L_{2}$-norm of the error. Since our bases are piecewise linear and the exact solution vector is 

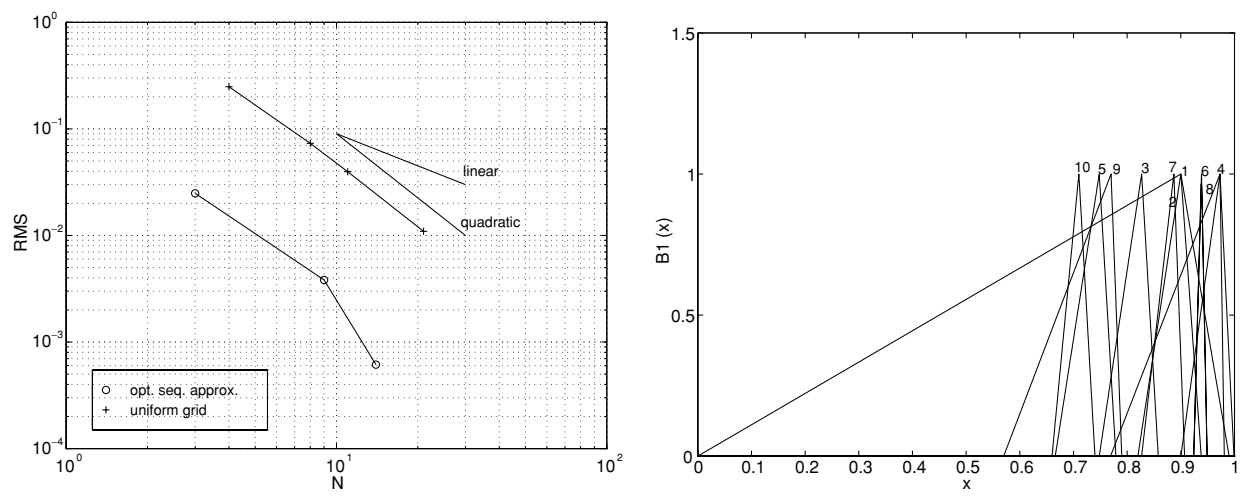

Figure 3. One-dimensional convection-diffusion. Convergence rates comparison (left) and distribution of the $N=10$ optimally selected basis functions (right).

exponential for our linear equation, we have $p=1, m=0$, and $s=\infty$ in Eq. (1). If the OIA does not adversely affect the optimal convergence rate of the bases then it should display at least a quadratic dependence on $N(\mu=2)$. This is confirmed in figure 3 (left). In addition, the comparison with uniform conventional finite elements in figure 3 (left) shows that the positive constant $C_{\delta}$ of Eq. (1) is substantially reduced by the OIA, which resembles the behavior of $r$-methods. Note that a greater than quadratic convergence is displayed at some steps of the OIA algorithm. This is partially explained by the "relocation" aspect of the OIA method, since it places the bases where they will reduce the magnitude of equation residual by the greatest amount.

\subsection{Computational cost}

At each step of the algorithm, the optimization problem (10) is solved using PDS. The computational cost depends on the search size, characterized by the number of simplex vertices (or pattern points) $d$, and the termination tolerance $t o l$; both are user-controlled parameters. PDS evaluates the objective function at $d$ vertices of a simplex in the hyperplane of dimension $n$, where $n$ is the number of optimization variables. After the completion of this process, the "best" vertex is chosen and compared to the previous iterate. If the difference lies between the user-defined tolerance $t o l$, the optimization process is terminated. In general, a small $d$ should be associated with a tight tolerance $t o l$, while a large $d$ should be combined with a relaxed tolerance $t o l$.

Table 1 summarizes the amount of computational work required by PDS for two combinations of parameter values: $\left(\mathrm{tol}=10^{-6}, d=100\right)$ and $\left(\mathrm{tol}=10^{-4}, d=1000\right)$. The number of iterations required by PDS at each step of the algorithm does not fluctuate significantly. However, it is observed that PDS requires fewer iterations at the first step of the algorithm. The number of required function evaluations is not very large, especially considering that an evaluation is quite inexpensive and that the computational work is distributed among four processors. In addition, the total number of solved optimization 
Table 1. Amount of computational work required by PDS for the linear convection-diffusion problem.

\begin{tabular}{cccc}
\hline $\begin{array}{l}\text { Algorithm } \\
\text { step }\end{array}$ & $\begin{array}{c}\text { No. of PDS } \\
\text { iterations }\end{array}$ & $\begin{array}{c}\text { Total no. of } \\
\text { function eval. }\end{array}$ & $\begin{array}{c}\text { Total no. of } \\
\text { constraint eval. }\end{array}$ \\
\hline $\begin{array}{c}d=100 \\
1\end{array}$ & 24 & tol $=10^{-6}$ & \\
8 & 18 & 836 & 2272 \\
16 & 15 & 1493 & 1536 \\
24 & 16 & 1307 & 1365 \\
1 & $d=1000$ & 1168 & 1335 \\
8 & 7 & 916 & 5344 \\
16 & 11 & 5721 & 6683 \\
24 & 12 & 6970 & 8719 \\
\hline
\end{tabular}

problems, which is equal to the number of algorithm steps, is small. The overall computational cost is therefore quite low. As expected, a low number of pattern points associated with a tighter tolerance requires more iterations but fewer function and constraint evaluations than a large number of pattern points associated with a relaxed tolerance.

\section{Nonlinear application: The velocity potential equation}

The derivation of natural variational principles for problems that are governed by nonlinear differential operators is generally a challenging task. However, there are applications of interest in fluid dynamics for which variational integrals have been proposed (Oden, 1992; Greenspan, 1965, 1967). For example, the problem of compressible inviscid fluid flow has been studied in its variational form extensively. Prozan (1982 a,b), discussed the existence of variational principles for unsteady conditions in one and two dimensions. Bateman (1930) derived two variational principles for isentropic conditions: a maximum principle defined in terms of the velocity potential and a minimum principle defined in terms of the stream function. Manwell (1980) proposed a variational integral for flow with shocks but does not present numerical results. His approach is similar to that presented earlier in Greenspan and Jain (1967), where a variational principle is extremized using finite differences. This variational principle is the same as that derived in Lush and Cherry (1956), which is based on the maximum principle from Bateman (1930) for steady conditions. The OIA method will be applied to the circular cylinder problem studied in Greenspan and Jain (1967).

The continuity and momentum equations governing steady, two-dimensional, isentropic, inviscid, compressible fluid flow past a fixed body can be combined by the introduction of a velocity potential $\varphi$ to the single scalar equation

$$
\left(c^{2}-\left(\frac{\partial \varphi}{\partial x}\right)^{2}\right) \frac{\partial^{2} \varphi}{\partial x^{2}}-2 \frac{\partial \varphi}{\partial x} \frac{\partial \varphi}{\partial y} \frac{\partial^{2} \varphi}{\partial x \partial y}+\left(c^{2}-\left(\frac{\partial \varphi}{\partial y}\right)^{2}\right) \frac{\partial^{2} \varphi}{\partial y^{2}}=0
$$


where $c$ is the local speed of sound. The associated boundary conditions are

$$
\frac{\partial \varphi}{\partial n}=0 \text { on the body and } \nabla \varphi=\left(V_{\infty}, 0\right)^{\mathrm{T}}=\text { constant far from the body, }
$$

where $n$ is the direction normal to the body surface and $V_{\infty}$ is the freestream velocity.

If the velocity potential $\varphi$ is defined in terms of some unknown auxiliary function $\chi$ according to

$$
\varphi=V_{\infty} x+V_{\infty} \chi,
$$

then the following functional to be maximized can be formulated in polar coordinates,

$$
E(\chi)=p_{\infty} \int_{\theta=0}^{2 \pi} \int_{r=1}^{\infty}\left(F-1+\gamma M_{\infty}^{2} H\right) r \mathrm{~d} r \mathrm{~d} \theta
$$

where

$$
F=\left(1-\frac{\gamma-1}{2} M_{\infty}^{2}\left(2 \cos (\theta) \frac{\partial \chi}{\partial r}-2 \frac{\sin (\theta)}{r} \frac{\partial \chi}{\partial \theta}+\left(\frac{\partial \chi}{\partial r}\right)^{2}+\frac{1}{r^{2}}\left(\frac{\partial \chi}{\partial \theta}\right)^{2}\right)\right)^{\frac{\gamma}{\gamma-1}}
$$

and

$$
H=\left(1-\frac{1}{r^{2}}\right) \frac{\partial \chi}{\partial r} \cos (\theta)-\left(1+\frac{1}{r^{2}}\right) \frac{\partial \chi}{\partial \theta} \frac{\sin (\theta)}{r} .
$$

In the above expressions $p$ is pressure, $M$ is the Mach number, $\gamma$ is the ratio of specific heats and the subscript $\infty$ denotes freestream conditions. The associated boundary conditions are

$$
\frac{\partial \chi}{\partial r}=-\cos (\theta) \text { at } r=1 \quad \text { and } \quad \chi=0 \text { far from the cylinder. }
$$

The variational principle of Eq. (14) is defined over an infinite domain. For the sake of comparison with the computations presented in Greenspan and Jain (1967), the computational domain has been modified to cover the area around a quarter of a unit cylinder $(\pi / 2 \leq \theta \leq \pi)$ with a radius that spans from the body surface $(r=1)$ to the far-field $(r=20)$. The boundary conditions for the new computational domain are

$$
\chi_{r}=-\cos (\theta) \text { at } r=1, \chi=0 \text { at } r=20 \text { and } \theta=\frac{\pi}{2}, \text { and } \chi_{\theta}=0 \text { at } \theta=\pi,
$$

where the subscripts denote partial differentiation.

\subsection{Numerical procedure}

The OIA method has been employed to approximate numerically the extremal function $\chi(r, \theta)$ that maximizes the variational principle. Utilizing the bilinear product $\Phi(r, \theta)=$ $\Phi(r) \Phi(\theta)$ for the representation of the two-dimensional basis function, the function $\chi(r, \theta)$ 
is approximated by the series expansion

$$
\chi_{N}^{a}(r, \theta)=\sum_{j=1}^{N} c_{j} \Phi_{j}\left(r, \bar{q}_{j}^{r}\right) \Phi_{j}\left(\theta, \bar{q}_{j}^{\theta}\right),
$$

where the basis functions $\Phi_{j}$ are $B_{1}$-splines with parameters $\bar{q}_{j}^{r}=\left(r_{M}, \Delta r_{l}, \Delta r_{r}\right)_{j}^{\mathrm{T}}$ and $\bar{q}_{j}^{\theta}=\left(\theta_{M}, \Delta \theta_{l}, \Delta \theta_{r}\right)_{j}^{\mathrm{T}}$. The $c_{j}$ are the associated expansion coefficients.

The presence of homogeneous Dirichlet boundary conditions on the boundaries $r=20$ and $\theta=\pi / 2$ facilitates the use of basis functions defined to vanish at the domain boundaries. The presence of Neumann boundary conditions on the boundaries $r=1$ and $\theta=\pi$, however, requires the extension of the computational domain in these two directions by the addition of a small fictitious domain characterized by the modifications of the lower and upper bounds in constraints (19) and (20), respectively. It is important to note that this is possible because the Neumann boundary conditions are naturally satisfied by the variational principle as shown in Kokkolaras (1997).

Only the partial derivatives of the function $\chi(r, \theta)$ appear in the functional $E$ of Eq. (14). They are approximated by

$$
\begin{aligned}
\chi_{r} \approx \frac{\partial \chi_{N}^{a}}{\partial r} & =\sum_{j=1}^{N} c_{j} \frac{\partial \Phi_{j}\left(r, \bar{q}_{j}^{r}\right)}{\partial r} \Phi_{j}\left(\theta, \bar{q}_{j}^{\theta}\right) \quad \text { and } \quad \chi_{\theta} \approx \frac{\partial \chi_{N}^{a}}{\partial \theta} \\
& =\sum_{j=1}^{N} c_{j} \Phi_{j}\left(r, \bar{q}_{j}^{r}\right) \frac{\partial \Phi_{j}\left(\theta, \bar{q}_{j}^{\theta}\right)}{\partial \theta} .
\end{aligned}
$$

These approximations are introduced into the variational integral to be maximized at each step of the algorithm. Due to the nonlinearity of the functional, the optimization problem cannot be decomposed into smaller problems as in the linear case. All seven optimization variables, represented by the vector $\bar{q}_{N}^{\prime}=\left(r_{M}, \Delta r_{l}, \Delta r_{r}, \theta_{M}, \Delta \theta_{l}, \Delta \theta_{r}, c\right)_{N}^{\mathrm{T}}$, are computed simultaneously by solving the optimization problem

$$
\begin{aligned}
& \max _{\bar{q}_{N}^{\prime}} E\left(\chi_{N}^{a}\right) \\
& \text { subject to } \quad 0.9 \leq r_{M}-\Delta r_{l} \leq r_{M} \leq r_{M}+\Delta r_{r} \leq 20
\end{aligned}
$$

and

$$
\frac{\pi}{2} \leq \theta_{M}-\Delta \theta_{l} \leq \theta_{M} \leq \theta_{M}+\Delta \theta_{r} \leq 1.1 \pi
$$

using PDS.

\subsection{Numerical results}

Numerical results are presented for transonic air flow around a quarter of a unit cylinder and compared to the results of Greenspan and Jain (1967). The following flow properties are 
estimated after solving for the function $\chi$ : the nondimensionalized speed of the fluid $V / V_{\infty}$, the nondimensionalized local speed of sound $c / V_{\infty}$, the nondimensionalized pressure $p / p_{\infty}$, and the pressure coefficient $C_{p}$.

Since the auxiliary function $\chi(\theta, r)$ is approximated by piecewise linear splines, its partial derivatives $\chi_{r}$ and $\chi_{\theta}$ required for the representation of the above mentioned quantities are approximated by piecewise constants. The results obtained from the partial derivatives of $\chi$ have been smoothed by a cubic polynomial fitted in the least-squares sense.

Numerical results were obtained for three test cases with freestream Mach numbers 0.4, 0.43 , and 0.46 in air (specific heat ratio $\gamma=1.405$ ). Greenspan and Jain (1967) reported results for $M_{\infty}=0.4$ and $M_{\infty}=0.43$, but their Newton-like algorithm failed to converge for freestream Mach numbers that were greater then 0.43. In their numerical test cases, $\Delta \theta=9^{\circ}$ and $\Delta r=0.1,0.15$, and 0.2 which result in finite difference grids of $11 \times 191=2101$, $11 \times 127=1397$, and $11 \times 96=1056$ nodes, respectively. Greenspan and Jain (1967) compared their results for $M_{\infty}=0.4$ to those presented in Lush and Cherry (1956) and Imai (1941).

The approximate function $\chi_{N}^{a}(r, \theta)$ is plotted on the left of figure 4 for $M_{\infty}=0.4$ using 31 optimally selected basis functions and associated coefficients. The function exhibits a steep gradient in the $r$-direction near the cylinder surface. A satisfactory level of accuracy is achieved considering the low number of optimal basis functions included in the series expansion.

The approximate solutions for $M_{\infty}=0.43$ and $M_{\infty}=0.46$ are similar to those of $M_{\infty}=$ 0.4 . The plot on the right of figure 4 and the plots in figure 5 present the approximation of $\chi_{N}^{a}(r, \theta)$ at different radii (on and near the cylinder surface) obtained for $M_{\infty}=0.46$ and $M_{\infty}=0.43$ using 41 and 30 optimal basis functions, respectively. It can be concluded that the agreement with the results presented in Greenspan and Jain (1967) is satisfactory. Moreover, the OIA method did not fail for $M_{\infty}>0.43$. The results presented for $M_{\infty}=0.46$ complete the ones reported in Greenspan and Jain (1967), Lush and Cherry (1956) and Imai (1941).
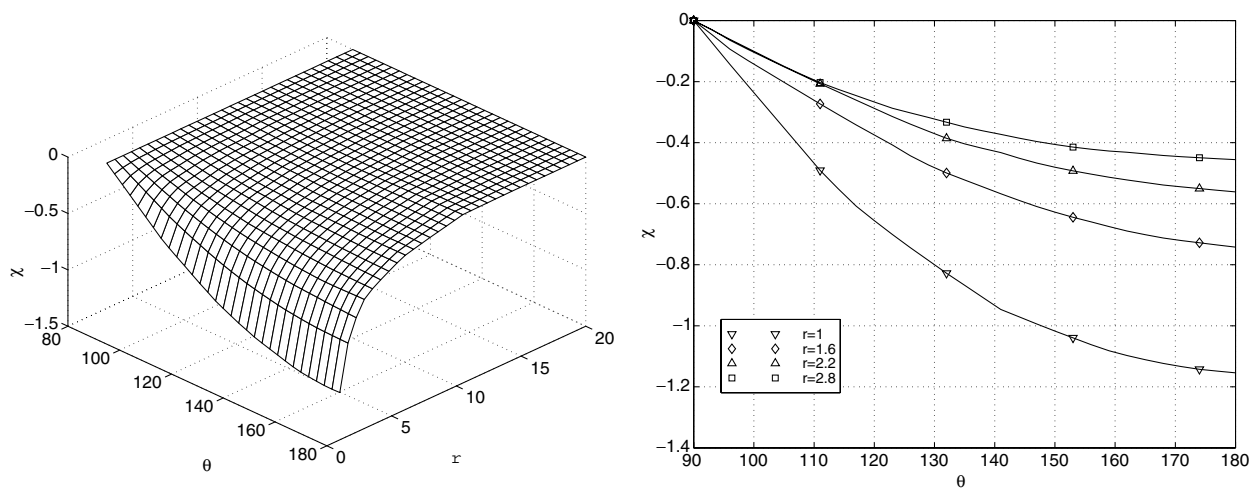

Figure 4. Two-dimensional, compressible, inviscid flow. The approximate velocity potential auxiliary function for $M_{\infty}=0.4$ using $N=31$ optimal basis functions (left) and the approximate velocity potential auxiliary function at different radii (on and near the cylinder surface) for $M_{\infty}=0.46$ using $N=41$ optimal basis functions (right). 

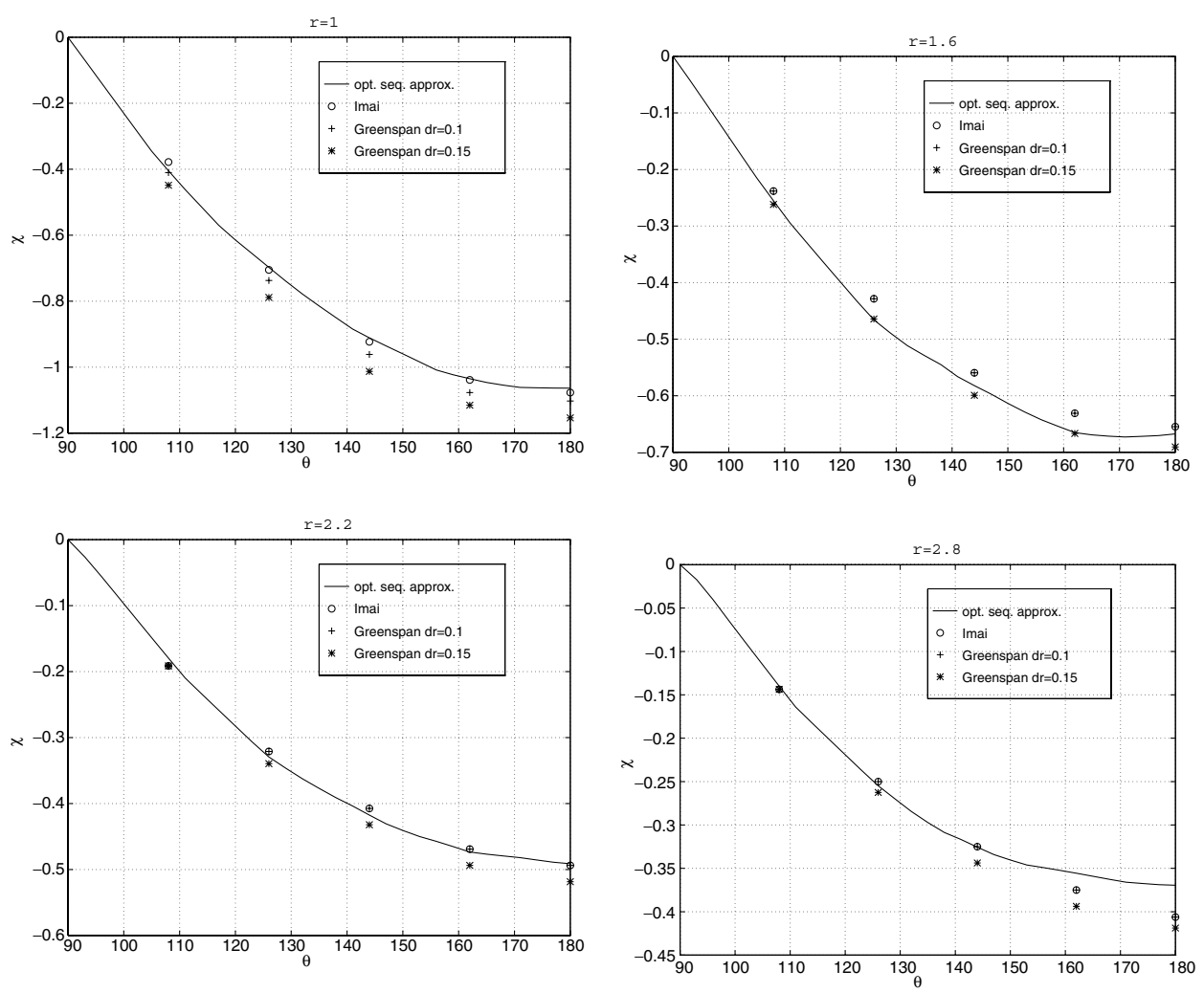

Figure 5. Two-dimensional, compressible, inviscid flow. The approximate velocity potential auxiliary function at different radii (on and near the cylinder surface) for $M_{\infty}=0.43$ using $N=30$ optimal basis functions.

Figure 6 depicts the nondimensionalized fluid velocity and sonic speed on the cylinder surface for the three test cases. The agreement with previously reported results is satisfactory. It can be seen how the flow just reaches sonic speed at $\theta=\pi / 2$ for $M_{\infty}=0.4$, while the flow becomes transonic for $M_{\infty}=0.43$ and $M_{\infty}=0.46$.

Finally, the pressure coefficient distributions on the cylinder surface for the three test cases are presented in figure 7. Again, the agreement with the available data validates the OIA method.

\subsection{Computational cost}

The amount of computational work required by PDS at each step of the algorithm is presented in Table 2 for $t o l=10^{-4}$ and $d=1120$. The number of iterations required by PDS to solve the optimization problem at each step of the algorithm does not exhibit extreme fluctuations. A larger number of function evaluations is required for the nonlinear application due to the increased size of the optimization problem. Evaluations are still quite inexpensive to perform however, and computational work is distributed among four processors. In 

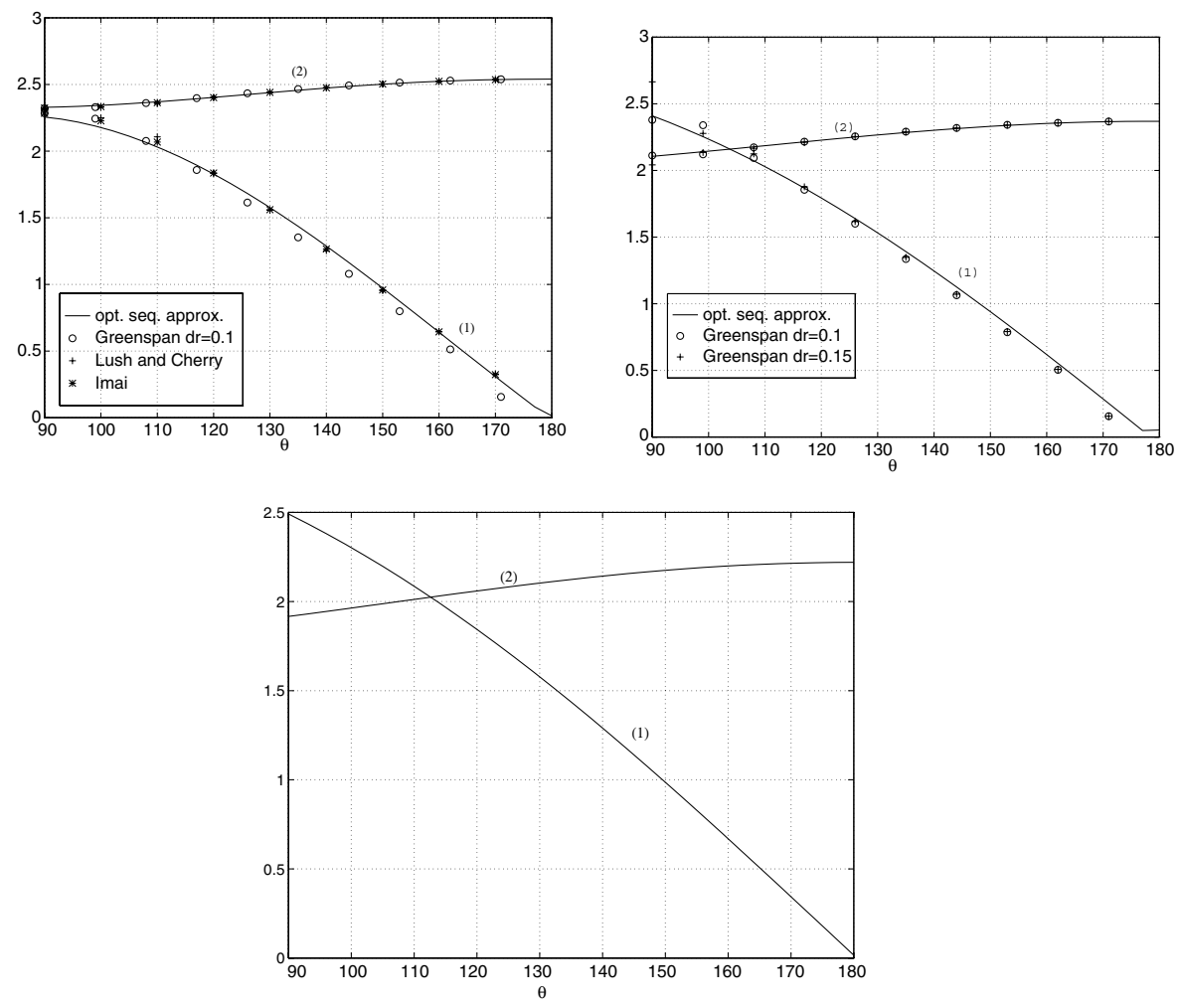

Figure 6. Two-dimensional, compressible, inviscid flow. Nondimensionalized fluid velocity (curve 1) and sonic speed on the cylinder surface (curve 2) for $M_{\infty}=0.4$ (top left), $M_{\infty}=0.43$ (top right), and $M_{\infty}=0.46$ (bottom).

addition, the total number of solved optimization problems, which is equal to the number of algorithm steps, has not increased substantially. The overall computational cost is therefore low.

\section{Challenges and opportunities}

It has been argued in the literature (Greenspan, 1965; Finlayson, 1972, p. 310) that the numerical approximation of the function that extremizes a variational principle is preferable to the numerical approximation of the function that satisfies the associated Euler-Lagrange equation. The performance of the proposed OIA method on problems where variational principles are available supports the above argument. Based on the presented examples, the OIA method seems to work very well on problems with linear (self- or nonself-adjoint) operators and on problems with nonlinear operators for which variational principles can be derived. The purpose of the OIA method is not to "compete" with traditional methods such as finite differences or finite elements, but to present an alternative that may work better 

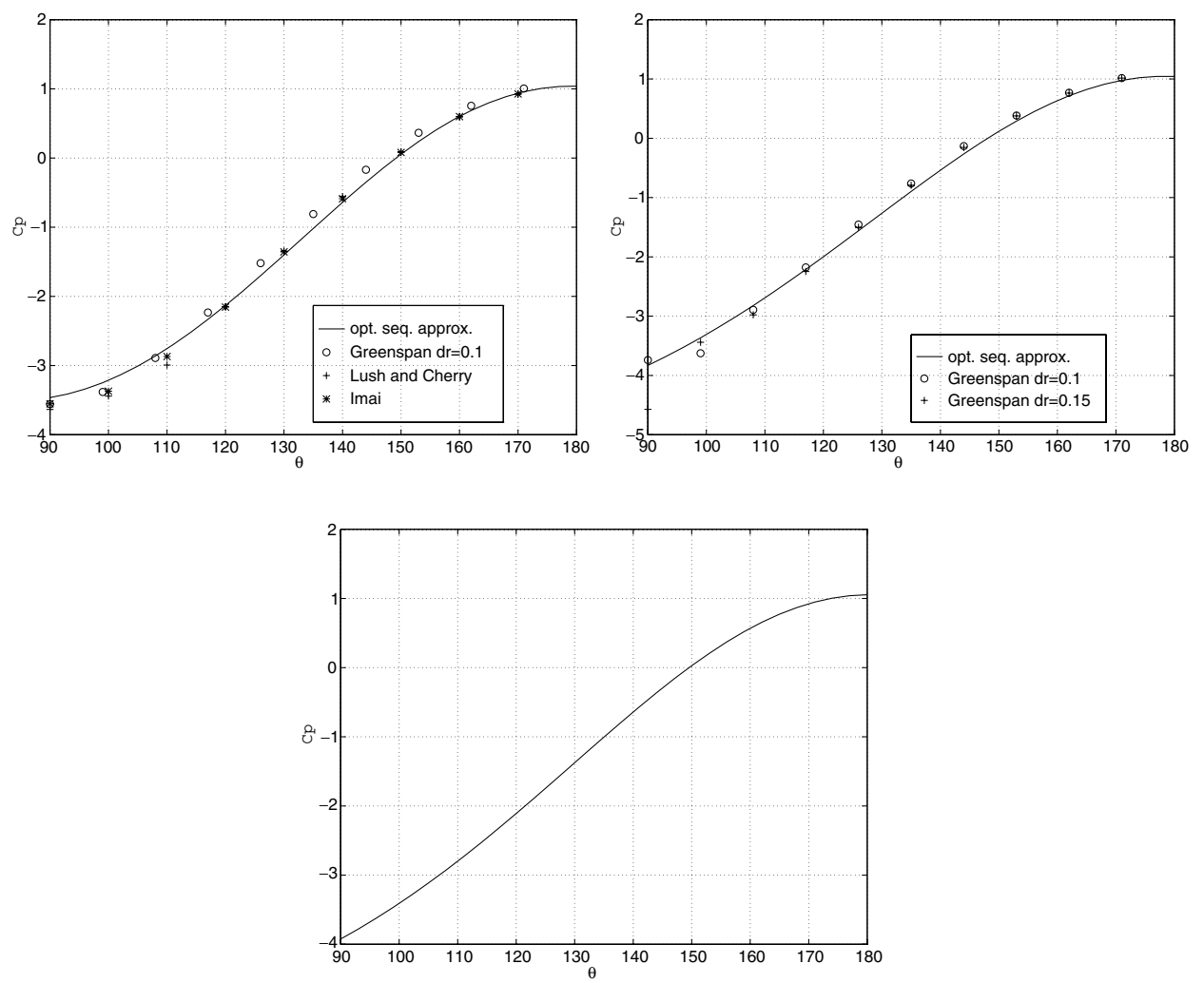

Figure 7. Two-dimensional, compressible, inviscid flow. Pressure coefficients distribution on the cylinder surface for $M_{\infty}=0.4$ (top left), $M_{\infty}=0.43$ (top right), and $M_{\infty}=0.46$ (bottom)

and/or more efficient relative to "simple" implementations of traditional methods in such problems. The point here is that "sophisticated" implementations of traditional methods may outperform the OIA method, however, they are more complicated and expensive.

As mentioned, the implementation of the OIA method in this paper is based on the utilization of variational principles. However, derivation of variational principles for all types of problems is far from straightforward; moreover, variational integrals may not always be associated with extremal functions. An alternative approach for defining the objective function in the optimization problem (3) is necessary.

The method of weighted residuals is a possible alternative, as discussed in Meade et al. (1997). For example, minimizing $\langle R, R\rangle$, where $R$ is the residual of a differential equation and $\langle\cdot\rangle$ is some appropriate inner product, corresponds to the well-known least-squares weighted residual method.

An alternative to the least-squares method can be found within the method of weighted residuals by considering the following geometric perspectives: if we assume that $\Psi_{N}$ is some function of the basis $\Phi_{N}$, then the parameters of the vector $\Psi_{N}$ can be selected such that it is as parallel as possible to the previous equation residual vector $R_{N-1}$. This is equivalent to 
Table 2. Amount of computational work required by PDS for the two-dimensional, compressible, inviscid flow problem for $d=1120$ and $t o l=10^{-4}$

\begin{tabular}{|c|c|c|c|c|}
\hline $\begin{array}{l}\text { Algorithm } \\
\text { step }\end{array}$ & $\begin{array}{c}\text { No. of PDS } \\
\text { iterations }\end{array}$ & $\begin{array}{c}\text { Total no. of } \\
\text { function eval. }\end{array}$ & $\begin{array}{c}\text { Total no. of } \\
\text { constraint eval. }\end{array}$ & $\begin{array}{c}\text { Functional } \\
\text { value }\end{array}$ \\
\hline \multicolumn{5}{|c|}{$M=0.4$} \\
\hline 1 & 21 & 6337 & 17202 & 0.053511 \\
\hline 10 & 16 & 11464 & 12155 & 0.093902 \\
\hline 20 & 42 & 33811 & 41611 & 0.095980 \\
\hline 30 & 17 & 13711 & 15000 & 0.096385 \\
\hline \multicolumn{5}{|c|}{$M=0.43$} \\
\hline 1 & 17 & 8014 & 12655 & 0.069409 \\
\hline 10 & 15 & 7941 & 13806 & 0.106047 \\
\hline 20 & 22 & 20452 & 21232 & 0.111336 \\
\hline 30 & 24 & 19443 & 24323 & 0.113558 \\
\hline \multicolumn{5}{|c|}{$M=0.46$} \\
\hline 1 & 17 & 7543 & 12654 & 0.080411 \\
\hline 10 & 31 & 23691 & 27128 & 0.128511 \\
\hline 20 & 44 & 28435 & 39596 & 0.143601 \\
\hline 30 & 24 & 23453 & 23643 & 0.149425 \\
\hline 40 & 37 & 36678 & 38842 & 0.150619 \\
\hline
\end{tabular}

maximizing $\left\langle R_{N-1}, \Psi_{N}\right\rangle^{2}$. The associated coefficient $c_{N}$ can be determined by forcing the residual $R_{N}$ to be as orthogonal as possible to $\Psi_{N}$. This is done by using the optimization variable $c_{N}$ to minimize $\left\langle R_{N}, \Psi_{N}\right\rangle^{2}$. The similarity of this technique to the Petrov-Galerkin method is apparent.

Thomson (1999) and Thomson et al. (2001) applied the OIA method to heat transfer problems successfully using functionals that were defined according to the above suggestions.

The OIA method may also be used in combination with mixed variable programming algorithms to solve problems with singular solutions. As discussed in Belytschko et al. (1996), the moving least-squares (MLS) meshless method employs predefined sets of basis functions. The associated coefficients are functions of the independent variables $\bar{x}$ and are determined by minimizing some appropriate criterion. When the problems have singular solutions, singular basis functions should be included in the predefined basis function sets. In fact, a user should be able to choose among many types of functions and their combinations: trigonometric, exponential, polynomial, or singular functions. The OIA method could be extended to also determine the optimal type of basis function to be included in the series expansion. A pattern search optimization algorithm has been developed recently for mixed variable programming (MVP) (Audet and Dennis, 2000). MVP has been applied to problems with categorical variables (Kokkolaras et al., 2001). Categorical variables are integer variables that cannot be relaxed to continuous variables during the optimization process since the objective function cannot be evaluated. The basis function type is such 
a categorical variable. Using MVP as the optimization algorithm instead of PDS, the OIA method could determine which basis function type to use at each step of the algorithm in addition to its optimal parameters and associated coefficient.

Treating complicated geometries is the major challenge faced by the proposed method. A possible remedy may be hybridization with other techniques. The computational domain could be partitioned, and the OIA method would work well for the part of the domain described by simple geometry while traditional techniques take care of the boundaries.

\section{Conclusions}

We present an optimal incremental approximation (OIA) method for the adaptive and meshless solution of problems for which variational principles are available. The proposed algorithm requires the solution of a nonlinear optimization problem at each of its steps. The variational principles associated with the differential equations and boundary conditions of the problems are utilized to define the objective function(al).

The OIA method is adaptive in nature although a grid is neither built nor adapted in the traditional sense. The computational overhead of the a posteriori error estimation and adaptive process, present in traditional adaptive and multigrid techniques, is avoided. The "grid", defined by the location of the optimally determined basis function parameters, evolves with the solution. In this sense, the method can be viewed as a meshless method. Complicated data structures are not required since systems of equations are neither assembled nor solved, and numerical stability issues are limited to the nonlinear optimization process. The algorithm can be initialized with an empty set of basis functions and utilize a broad class of functions.

The OIA method has been applied successfully to a linear nonself-adjoint problem and a nonlinear problem associated with general boundary conditions. The OIA method exploits its adaptive nature in problems whose solutions exhibit large gradients. The accuracy of the obtained results is satisfactory considering the low number and order of the optimal basis functions used in the series expansion that approximates the solution. As shown in the case of the linear application, the OIA method overcomes certain difficulties faced by the finite differences methods when the latter does not employ an upwind scheme. In addition, although we cannot estimate convergence rates theoretically at this point, the convergence rate obtained in practice for the linear application seems to be superior to that of the Bubnov-Galerkin technique using a uniform grid. Of course, upwind schemes can be employed in finite differences, and the finite element method could be utilized with higher order basis functions and more sophisticated meshes, in which cases they would outperform the OIA method. However, the purpose of the OIA method is to present an attractive alternative for simple (adaptive but meshless, using linear basis functions) and efficient (low computational cost without deteriorating accuracy) solution of problems that can be represented by variational principles.

Any nonlinear programming algorithm can be employed for the solution of the optimization problems. However, using the parallel direct search (PDS) algorithm, which features concurrent function evaluations, we keep the computational cost low. Readily parameterized low order functions and their bilinear products have been used to ensure simplicity 
and computational efficiency. The computational work required by PDS does not increase as the algorithm advances.

The implementation of the OIA method in this paper is based on the utilization of natural and contrived variational principles. We propose alternative formulations for problems where variational principles may not be available and point to references of recent work in which these formulations were used successfully in the solution of heat transfer problems. Finally, we discuss a possible extension of the method using mixed variable programming algorithms to also determine the type of basis function to be used in the series expansion.

\section{Acknowledgments}

This work was supported under NASA grant no. CRA2-35504 and ONR grant no. N00014-95-1-0741. Access to the computing facilities of the Center for Research on Parallel Computation (CRPC) at Rice University and the assistance of David Serafini with the optimal utilization of the PDS software are greatly appreciated. The authors would like to thank the anonymous referees for their constructive comments.

\section{References}

C. Audet and J. E. Dennis, Jr., "Pattern search algorithms for mixed variable programming," SIAM Journal on Optimization vol. 11, no. 3, pp. 573-594, 2000.

A. R. Barron, "Universal approximation bounds for superpositions of a sigmoidal function," IEEE Transactions on Information Theory vol. 39, no. 3, pp. 930-945, 1993.

H. Bateman, "Irrotational motion of a compressible inviscid fluid," Proc. Nat. Acad. Sci. vol. 16, pp. 816-825, 1930.

T. Belytschko, Y. Krongauz, D. Organ, M. Fleming, and P. Krysl, "Meshless methods: An overview and recent developments," Computer Methods in Applied Mechanics and Engineering vol. 139, pp. 3-47, 1996.

L. Demkowicz and J. T. Oden, "On a mesh optimization method based on a minimization of interpolation error," Int. J. Engng. Sci. vol. 24, no. 1, pp. 55-68, 1986.

J. E. Dennis, Jr. and V. Torczon, "Direct search methods on parallel machines," SIAM Journal of Optimization vol. 1, no. 4, pp. 448-474, 1991.

A. R. Diaz, N. Kikuchi, and J. E. Taylor, "A method of grid optimization for finite element methods," Computer Methods in Applied Mechanics and Engineering vol. 41, pp. 29-45, 1983.

P. R. Eiseman, Y. K. Choo, and R. E. Smith, "Algebraic grid generation with control points," in Finite Elements in Fluids, T. J. Chung (Ed.), vol. 8, Hemisphere Publ. Corp., 1992.

B. A. Finlayson, The Method of Weighted Residuals and Variational Principles, Academic Press, 1972.

C. A. J. Fletcher, Computational Galerkin Methods, Springer Verlag, 1982.

C. A. J. Fletcher, Computational Techniques for Fluid Dynamics, vol. 1, Springer Verlag, 1988.

D. Greenspan, "On approximating extremals of functionals: I the method and examples for boundary value problems," Bull. Int. Comp. Centre vol. 4, no. 2, pp. 99-120, 1965. University of Rome.

D. Greenspan, "On approximating extremals of functionals: II theory and generalizations related to boundary value problems for nonlinear differential equations," Int. J. Engng. Sci. vol. 5, pp. 571-588, 1967.

D. Greenspan and P. C. Jain, "Application of a method for approximating extremals of functionals to compressible flow," Mathematical Analysis and Applications vol. 18, no. 1, pp. 85-111, April 1967. Also available as technical report TR 597 from the Mathematical Research Center of the University of Wisconsin, Jan. 1966.

O. Hassan, K. Morgan, and J. Peraire, "Finite-element solution scheme for compressible viscous flow," in Finite Elements in Fluids, T. J. Chung (Ed.), vol. 8. Hemisphere Publ. Corp., 1992. 
I. Imai, "On the flow of a compressible fluid past a circular cylinder," Proc. Phys. Math. Soc. Japan vol. 23, ser. 3, pp. 180-193, 1941.

C. Johnson, Numerical Solution of Partial Differential Equations by the Finite Element Method, Cambridge University Press, 1990.

L. K. Jones, "Constructive approximations for neural networks by sigmoidal functions," Proceedings of the IEEE vol. 78, no. 10, pp. 1586-1589, 1990.

L. K. Jones, "A simple lemma on greedy approximation in Hilbert space and convergence rates for projection pursuit regression and neural network training," The Annals of Statistics vol. 20, no. 1, pp. 608-613, 1992.

M. Kokkolaras, Utilizing Parallel Optimization in Computational Fluid Dynamics, PhD thesis, Rice University, Houston, Texas, 1997.

M. Kokkolaras, C. Audet, and J. E. Dennis, Jr, "Mixed variable optimization of the number and composition of heat intercepts in a thermal insulation system," Optimization and Engineering vol. 2, no. 1, pp. 5-29, 2001.

P. E. Lush and T. M. Cherry, "The variational method in hydrodynamics," J. Mech. Appl. Math. vol. 9, pp. 6-21, 1956.

A. R. Manwell, "A variational principle for steady homenergic compressible flow with finite shocks," Wave Motion vol. 2, no. 1, pp. 83-95, 1980.

A. J. Meade, Jr., M. Kokkolaras, and B. A. Zeldin, "Sequential function approximation for the solution of differential equations," Communications in Numerical Methods in Engineering vol. 13, pp. 977-986, 1997.

J. T. Oden, "Adaptive methods in computational fluid dynamics," in Finite Elements in Fluids, T. J. Chung (Ed.), vol. 8. Hemisphere Publ. Corp., 1992.

E. R. Oliveira, "Optimization of finite element solutions," in Proc. Third Conf. Matrix Methods in Struct. Mech., Ohio, 1971. Air Force Flight Dynamics Laboratory, Wright-Patterson AFB. AFFDL TR 71-160.

R. J. Prozan, "A variational principle for compressible fluid mechanics: Discussion of the multi-dimensional theory," Technical Report 3614, NASA, 1982a.

R. J. Prozan, "A variational principle for compressible fluid mechanics: Discussion of the one-dimensional theory," Technical Report 3526, NASA, 1982b.

D. L. Thomson, Sequential Function Approximation of the Radiative Transfer Equation, PhD thesis, Rice University, Houston, Texas, 1999.

D. L. Thomson, A. J. Meade, and Y. Bayazitoglu, "Solution of the radiative transfer equation in discrete ordinate form by sequential function approximation," International Journal of Thermal Sciences vol. 40, no. 6, pp. 517$527,2001$.

O. C. Zienkiewicz and R. L. Taylor, The Finite Element Method, McGraw-Hill, 1989. 